\title{
Ambulatory Surgical Procedure
}

National Cancer Institute

\section{Source}

National Cancer Institute. Ambulatory Surgical Procedure. NCI Thesaurus. Code C51997.

A surgical procedure performed on an outpatient basis. It may be hospital-based or performed in an office or surgicenter. 